\title{
Reasoning processes in clinical reasoning: from the perspective of cognitive psychology
}

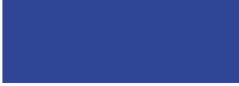

\author{
Hyoung Seok Shin
}

Department of Medical Education, Korea University College of Medicine, Seoul, Korea

Clinical reasoning is considered a crucial concept in reaching medical decisions. This paper reviews the reasoning processes involved in clinical reasoning from the perspective of cognitive psychology. To properly use clinical reasoning, one requires not only domain knowledge but also structural knowledge, such as critical thinking skills. In this paper, two types of reasoning process required for critical thinking are discussed: inductive and deductive. Inductive and deductive reasoning processes have different features and are generally appropriate for different types of tasks. Numerous studies have suggested that experts tend to use inductive reasoning while novices tend to use deductive reasoning. However, even experts sometimes use deductive reasoning when facing challenging and unfamiliar problems. In clinical reasoning, expert physicians generally use inductive reasoning with a holistic viewpoint based on a full understanding of content knowledge in most cases. Such a problem-solving process appears as a type of recognition-primed decision making only in experienced physicians' clinical reasoning. However, they also use deductive reasoning when distinct patterns of illness are not recognized. Therefore, medical schools should pursue problem-based learning by providing students with various opportunities to develop the critical thinking skills required for problem solving in a holistic manner.

Key Words: Clinical reasoning, Inductive reasoning, Deductive reasoning, Critical thinking skills

\section{Introduction}

It is hard to describe clinical reasoning in a sentence, because it has been studied by a number of researchers from various perspectives, such as medical education, cognitive psychology, clinical psychology, and so forth, and they have failed to reach an agreement on its basic characteristics [1]. Accordingly, clinical reasoning has been defined in various ways. Some researchers defined clinical reasoning as a crucial skill or ability that all physicians should have for their clinical decision making, regardless of their area of expertise [2,3]. Others focused more on the processes of clinical reasoning; thus, they defined it as a complex process of identifying the clinical issues to propose a treatment plan [4-6]. However, these definitions are not so different. Taking this into account, it can be concluded that clinical reasoning is used to analyze patients' status and arrive at a medical decision so that doctors can provide the proper medical treatment.

In reality, properly working clinical reasoning requires three domains of knowledge: diagnostic knowledge, etiological knowledge, and treatment knowledge [6].
Received: October 21, 2019 • Revised: October 21, 2019 • Accepted: October 30, 2019 Corresponding Author: Hyoung Seok Shin (https://orcid.org/0000-0002-7785-4755) Department of Medical Education, Korea University College of Medicine, 73 Goryeodae-ro, Seongbuk-gu, Seoul 02841, Korea

Tel: +82.2.2286.1206 Fax: +82.2.928.1647 email: mozart1980@korea.ac.kr
Korean J Med Educ 2019 Dec; 31(4): 299-308.

https://doi.org/10.3946/kjme.2019.140

eISSN: 2005-7288

(C) The Korean Society of Medical Education. All rights reserved. This is an open-access article distributed under the terms of the Creative Commons Attribution Non-Commercial License (http:// creativecommons.org/licenses/by-nc/3.0/), which permits unrestricted non-commercial use, distribution, and reproduction in any medium, provided the original work is properly cited. 
From the perspective of cognitive psychology, structural knowledge is needed to integrate domain knowledge and find solutions based on the learner's prior knowledge and experience [7], and structural knowledge can be constructed as a form of mental model by understanding the relations between the interconnected factors involved in clinical issues $[8,9]$. In this cognitive process, critical thinking skills such as causal reasoning and systems thinking can play a pivotal role in developing deeper understanding of given problem situations. Causal reasoning is the ability to identify causal relationships between sets of causes and effects [10]. Causality often involves a series or chain of events that can be used to infer or predict the effects and consequences of a particular cause [10-13]. Systems thinking is a thinking paradigm or conceptual framework where understanding is defined in terms of how well one is able to break a complex system down into its component parts [14,15]. It is based on the premise that a system involves causality between factors that are parts of the system as a whole [14]. Systems thinking is a process for achieving a deeper understanding of complex phenomena that are composed of components that are causally interrelated [14-16]. As a result, causal reasoning and systems thinking are skills that can help people to better understand complex phenomena in order to arrive at effective and targeted solutions that address the root causes of complex problems [10,12,15].

If cognitive skills work properly, one can make correct decisions all of the time. However, human reasoning is not always logical, and people often make mistakes in their reasoning. The more difficult the problems with which they are presented, the more likely they are to choose wrong answers that are produced by errors or flaws in the reasoning process [17,18]. Individual differences in reasoning skills - such as systems thinking, causal reasoning, and thinking processes may influence and explain observed differences in their understanding. Therefore, to better assist learners in solving problems, instructors should focus more on facilitating the reasoning skills required to solve given problems successfully.

In this review paper, the author focuses on the reasoning processes involved in clinical reasoning, given that clinical reasoning is considered as a sort of problem-solving process. Therefore, this paper introduces concepts related to the reasoning processes involved in clinical reasoning and their influences on novices and experts in the field of medical education from the perspective of cognitive psychology. Then, based on the contents discussed, the author will be able to propose specific instructional strategies associated with reasoning processes to improve medical students' reasoning skills to enhance their clinical reasoning.

\section{Concepts and nature of reasoning processes}

Generally, reasoning processes can be categorized into two types: inductive/forward and deductive/backward [19]. In an inductive reasoning process, one observes several individual facts first, then makes a conclusion about a premise or principle based on these facts. Yet there may be the possibility that a conclusion is not true even though a premise or principle in support of that conclusion is true, because the conclusion is generalized from the facts observed by the learner, but the learner does not observe all relevant examples [20].

In general, in a deductive reasoning process, according to Johnson-Laird [20], one establishes a mental model or a set of models to solve given problems considering general knowledge and principles based on a solid foundation. Then, one makes a conclusion or finds a 
solution based on the mental model or set of models. To verify a mental model, one needs to check the validity of the conclusions or solutions by searching for counterexamples. If one cannot find any counterexamples, the conclusions can be accepted as true and the solutions as valid. Consequently, the initial mental model or set of models can be used for deductive reasoning.

Anderson [17] proposed three different ways of solving complex problems: means-ends analysis, working backward, and planning by simplification. A means-ends analysis is a process that gets rid of differences between the current state and the ideal state in order to determine sub-goals in solving problems, and the process can be repeated until the major goal is achieved [21-23]. It can be considered an inductive reasoning process, because the distinct feature of means-ends analysis where it achieves sub-goals in consecutive order is similar to inductive reasoning. Working backward is addressed as an opposite concept to means-ends analysis [17], because it needs to set up a desired result to find causes by measuring the gap between the current state and the ideal state; then, this process is repeated until the root causes of a problem are identified. According to Anderson [17], means-ends analysis (inductive reasoning) is more useful in finding a solution quickly when a limited number of options are given or many sub-goals should be achieved for the major goal; whereas working backward (deductive reasoning) spends more time removing wrong answers or inferences to find the root causes of a problem. In conclusion, inductive and deductive reasoning processes have different features and can play different roles in solving complex problems.

\section{The use of reasoning processes}

A number of researchers across different fields have used inductive and deductive approaches as reasoning processes to solve complex problems or complete tasks. For example, Scavarda et al. [24] used both approaches in their study to collect qualitative data through interviews with experts, and they found that experts with a deductive approach used a top-down approach and those with an inductive approach used a bottom-up approach to solve a given problem. In a study of Overmars et al. [25], the results showed that a deductive approach explicitly illustrated causal relations and processes in 39 geographic contexts and it was appropriate for evaluating various possible scenarios; whereas an inductive approach presented associations that did not guarantee causality and was more useful for identifying relatively detailed changes.

Sharma et al. [26] found that inductive or deductive approaches can both be useful depending on the characteristics of the tasks and resources available to solve problems. An inductive approach is considered a data-driven approach, which is a way to find possible outcomes based on rules detected from undoubted facts [26]. Therefore, if there is a lot of available data and an output hypothesis, then it is effective to use an inductive approach to discover solutions or unexpected and interesting findings [26,27]. An inductive approach makes it possible to directly reach conclusions via thorough reasoning that involves the following procedures: (1) recognize, (2) select, and (3) act [28]. These procedures are recurrent, but one cannot know how long they should be continued to complete a task, because a goal is not specified [26]. Consequently, an inductive approach is useful when analyzing an unstructured data set or system [29]. 
Table 1. Features of Inductive and Deductive Reasoning Processes

\begin{tabular}{lll}
\hline & \multicolumn{1}{c}{ Inductive reasoning } & Deductive reasoning \\
\hline Information processing & Bottom-up & Top-down \\
Reasoning direction & Forward & Backward \\
Data processing strategies & Data driven & Goal driven \\
Appropriate data type & Unstructured data & Well-structured data \\
Appropriate task type & Exploratory tasks & Diagnostic and classification tasks \\
How to reach a conclusion & Collecting evidence from cases then building a general & Setting up a hypothesis then reaching a logical \\
& principle (specific cases $\rightarrow$ general principle) & conclusion (hypothesis $\rightarrow$ cases) \\
Validity of conclusion & A conclusion can be false even if all the premises & A conclusion cannot be false if the premises are true \\
& are true & \\
Required ability & The ability to recognize meaningful patterns and & The ability to logically reason \\
& connections & \\
Purpose of reasoning & Formation of hypotheses and theories & Prediction of consequences \\
\hline
\end{tabular}

The classification according to the reasoning processes in the table is dichotomous, but they do not always follow this classification absolutely. This means that each reasoning process shows such tendencies.

On the other hand, a deductive approach sets up a desired goal first, then finds a supporting basis - such as information and rules for the goals [26]. For this, a backward approach, which is considered deductive reasoning, gradually gets rid of things proved unnecessary for achieving the goal while reasoning; therefore, it is regarded as a goal-driven approach [28]. If the output hypothesis is limited and it is necessary to find supporting facts from data, then a deductive approach would be effective [26,28]. This implies that a deductive approach is more appropriate when a system or phenomenon is well-structured and relationships between the components are clearly present [29]. Table 1 shows a summary of the features and differences of the inductive and deductive reasoning processes.

Considering the attributes of the two reasoning processes, an inductive approach is effective for exploratory tasks that do not have distinct goals for example, planning, design, process monitoring, and so on, while a deductive approach is more useful for diagnostic and classification tasks [26]. In addition, an inductive approach is more useful for discovering solutions from an unstructured system. On the other hand, a deductive approach can be better used to identify root causes in a well-structured context. While both reasoning approaches are useful in particular contexts, it can be suggested that inductive reasoning is more appropriate than deductive reasoning in clinical situations, which focus on diagnosis and treatment of diseases rather than on finding their causes.

\section{Reasoning processes by novices and experts}

As mentioned above, which reasoning process is more effective for reaching conclusions can be generally determined depending on the context and purpose of the problem solving. In reality, however, learners' choices are not always consistent with this suggestion, because they are affected not only by the problem itself, but also by the learner. Assuming that learners or individuals can be categorized into two types, novices and experts, based on their level of prior knowledge and structural knowledge, much research has shown that novices and experts use a different reasoning process for problem solving. For example, in a study of Eseryel et al. [30], novice instructional designers who possessed theoretical knowledge but little experience showed different patterns of ill-structured problem solving compared to 
experts with real-life experience. Given that each learner has a different level of prior knowledge relating to particular topics and critical thinking skills, selecting the proper reasoning process for each problem is quite complex. This section focuses on which reasoning process an individual uses depending on their content and structural knowledge.

Numerous studies have examined which reasoning processes are used by experts, who have sufficient content and structural knowledge, and novices, who have little content and structural knowledge, for problem solving. The result of a study of Hong et al. [31] showed that children generally performed better when using cause-effect inferences (inductive approach) than effectcause inferences (considered a deductive approach). According to Anderson [17], people are faced with some difficulties when they solve problems using induction. The first difficulty is in formulating proper hypotheses and the second is that people do not know how to interpret negative evidence when it is given and reach a conclusion based on that evidence [17]. Nevertheless, most students use a type of inductive reasoning to solve problems that they have not previously faced [32]. Taken together, the studies suggest that novices generally prefer an inductive approach to a deductive approach for solving problems because they may feel comfortable and natural using an inductive approach but tend to experience difficulties during problem-solving processes. From these findings, it can be concluded that novices are more likely to use inductive reasoning, but it is not always productive.

Nevertheless, there is still a controversy about which reasoning processes are used by experts or novices [33]. For example, experts in specific domains use an inductive approach to solving problems, but novices, who have a lower level of prior knowledge in specific domains, tend to use a deductive approach [23]. In contrast, according to Smith [34], studies in which more familiar problems were used concluded that experts preferred an inductive approach, whereas in studies that employed relatively unfamiliar problems that required more time and effort to solve, experts tended to prefer a deductive approach. In line with this finding, in solving physics problems, experts mostly used inductive reasoning that was faster and had fewer errors for problem solving only when they encountered easy or familiar problems where they could gain a full understanding of the situation quickly, but novices took more time to deductively reason by planning and solving each step in the process of problem solving [35].

Assuming that an individual's prior knowledge consists of content knowledge such as knowledge of specific domains as well as structural knowledge such as the critical thinking skills required for problem solving in the relevant field, it seems experts use an inductive approach when faced with relatively easy or familiar problems; while a deductive approach is used for relatively challenging, unfamiliar, or complex problems. In the case of novices, it may be better to use deductive reasoning for problem solving considering that they have a lower level of prior knowledge and that even experts use deductive reasoning to solve complex problems.

\section{Inductive and deductive reasoning in clinical reasoning}

In medicine, concepts of inductive and deductive reasoning apply to gathering appropriate information and making a clinical diagnosis considering that the medical treatment process is a form of problem solving. Inductive reasoning is used to make a diagnosis by starting with an analysis of observed clinical data $[36,37]$. Inductive reasoning is considered as scheme- 
inductive problem solving in medicine [36], because in inductive reasoning, one first constructs his/her scheme (also considered a mental model) based on one's experiences and knowledge. It is generally used for a clinical presentation-based model, which has been most recently applied to medical education [38].

In contrast, deductive reasoning entails making a clinical diagnosis by testing hypotheses based on systematically collected data [39]. Deductive reasoning is considered an information-gathering method, because one constructs a hypothesis first then finds supporting or refuting facts from data [36,40]. It has been mostly used for discipline-based, system-based, and case-based models in medical education [38].

\section{Inductive and deductive reasoning by novice and expert physicians}

A growing body of research explores which reasoning processes are mainly used by novices and experts in clinical reasoning. Novice physicians generally use deductive reasoning, because limited knowledge restricts them from using deductive reasoning [1,38]. Also, it is hard to consider deductive reasoning as an approach generally used by experts, since they do not repeatedly test a hypothesis based on limited knowledge in order to move on to the next stage in the process of problem solving [38]. Therefore, it seems that deductive reasoning is generally used by novices, while inductive reasoning is used by expert physicians in general. However, this may be too conclusive and needs to be further examined in the context of clinical reasoning. In clinical reasoning, inductive reasoning is more intuitive and requires a holistic view based on a full understanding of content knowledge, including declarative and procedural knowledge, but also structural knowledge; thus, it occurs only when physicians' knowledge structures of given problems are highly organized [38]. Expert physicians recognize particular patterns of symptoms through repeated application of deductive reasoning, and the pattern recognition process makes it possible for them to apply inductive reasoning when diagnosing patients [10]. As experts automate a number of cognitive sequences required for problem solving in their own fields [35], expert physicians automatically make appropriate diagnoses following a process of clinical reasoning when they encounter patients who have familiar or typical diseases. Such a process of problem solving is called recognition-primed decision making (RPDM) [41,42]. It is a process of finding appropriate solutions to ill-structured problems in a limited timeframe [10]. In RPDM, expert physicians are aware of what actions should be taken when faced with particular situations based on hundreds of prior experiences [10]. These prior experiences are called illness scripts in diagnostic medicine [10], and this is a concept similar to a mental model or schema in problem solving.

However, expert physicians do not always use inductive reasoning in their clinical reasoning. Jonassen [10] categorized RPDM into three forms of variations in problem solving by experts, and the first form of variation is the simplest and easiest one based on inductive reasoning, as mentioned above. The second type of variation occurs when an encountered problem is somewhat atypical [10]. Even expert physicians are not always faced with familiar or typical diseases when treating patients. Expert physicians' RPDM does not work automatically when faced with atypical symptoms, because they do not have sufficient experiences relevant to the atypical symptoms. In this case, it can be said that they have weak illness scripts or mental models of the given symptoms. In the second variation, experts need 
more information and will attempt to connect it to their prior knowledge and experiences [10]. Deductive reasoning is involved in this process so that problem solvers can test their hypotheses in order to find new patterns and construct new mental models based on the newly collected data and previous experiences. The third variation of RPDM is when expert physicians have no previous experience or prior knowledge of given problem situations; in other words, no illness script or mental model [10]. Jonassen [10] argued that a mental simulation is conducted to predict the consequences of various actions by experts in the third variation. This process inevitably involves repetitive deductive reasoning to test a larger number of hypotheses when making a diagnosis.

Similarly, from the perspective of dual process theory as a decision-making process, decision making is classified into two approaches based on the reasoning style: type 1 and type 2 (or system 1 and system 2) [43,44]. According to Croskerry [44], the type 1 decision-making process is intuitive and based on experiential-inductive reasoning, while type 2 is an analytical and hypotheticodeductive decision-making process [44,45]. A feature that distinguishes the two processes is whether a physician who encounters a patient's symptoms succeeds in pattern recognition. If a physician recognizes prominent features of the visual presentation of illness, type 1 processes (or system 1) are operated automatically, whereas type 2 (or system 2) processes work if any distinct feature of illness presentation is not recognized [44].

Only experienced expert physicians can use RPDM $[10,46]$ or type 1 and 2 processes [43], because it can occur solely based on various experiences and a wide range of prior knowledge that can be gained as a result of a huge amount of deductive reasoning since they were novices. Consequently, it can be concluded that expert physicians generally use more inductive reasoning when they automatically recognize key patterns of given problems or symptoms, while sometimes they also use deductive reasoning when they additionally need processes of hypothesis testing to recognize new patterns of symptoms.

\section{Conclusion}

From the perspective of cognitive processes, clinical reasoning is considered as one of the decision-making processes that finds the best solutions to patients' illnesses. As a form of decision making for problem solving, two reasoning processes have been considered: inductive and deductive reasoning. Deductive reasoning can be used to make a diagnosis if physicians have insufficient knowledge, sufficient time, and the ability to analyze the current status of their patients. However, in reality, it is inefficient to conduct thorough deductive reasoning at each stage of clinical reasoning because only a limited amount of time is allowed for both physicians and patients to reach a conclusion in most cases. A few researchers have suggested that using deductive reasoning is more likely to result in diagnostic errors than inductive reasoning, because evidence-based research, such as deductive reasoning, focuses mainly on available and observable evidence and rules out the possibility of any other possible factors influencing the patient's symptoms [37,38]. However, when a physician encounters unfamiliar symptom and the degree of uncertainty is high, deductive reasoning is required to reach the correct diagnosis through analytical and slow diagnostic processes by collecting data from resources [44]. Taken together, in order to make the most of a limited timeframe and reduce diagnostic errors, physicians should be encouraged to use inductive reasoning 
in their clinical reasoning as far as possible given that patterns of illness presentation are recognized.

Unfortunately, it is not always easy for novice physicians to apply inductive or deductive reasoning in all cases. Expert physicians have sufficient capabilities to use both inductive and deductive reasoning and can also automate their clinical reasoning based on inductive reasoning, because they have already gathered the wide range of experiences and knowledge required to diagnose various symptoms. Novice physicians should make a greater effort to use inductive reasoning when making diagnoses; however, it takes experiencing countless deductive reasoning processes to structure various illness scripts or strong mental models until they reach a professional level. As a result, teaching not only clinical reasoning as a whole process but also the critical thinking skills required for clinical reasoning is important in medical schools [47]. For this, medical schools should pursue problem-based learning by providing students with various opportunities to gain content knowledge as well as develop the critical thinking skills -such as data analysis skills, metacognitive skills, causal reasoning, systems thinking, and so forth-required for problem solving in a holistic manner so that they can improve their reasoning skills and freely use both inductive and deductive approaches in any context. Further studies will be reviewed to provide detailed guidelines or teaching tips on how to develop medical students' critical thinking skills.

ORCID:

Hyoung Seok Shin: https://orcid.org/0000-0002-7785-4755

Acknowledgements: None.

Funding: None.

Conflicts of interest: No potential conflict of interest relevant to this article was reported.
Author contributions: All work was done by HS.

\section{References}

1. Norman G. Research in clinical reasoning: past history and current trends. Med Educ. 2005;39(4):418-427.

2. Cutrer WB, Sullivan WM, Fleming AE. Educational strategies for improving clinical reasoning. Curr Probl Pediatr Adolesc Health Care. 2013;43(9):248-257.

3. Eva KW. What every teacher needs to know about clinical reasoning. Med Educ. 2005;39(1):98-106.

4. Higgs J, Jones M. Clinical decision-making and multiple problem spaces. In: Higgs J, Jones $\mathrm{M}$, eds. Clinical Reasoning in the Health Professions. 3rd ed. Oxford, UK: Butterworth-Heineman Ltd.; 2008:3-17.

5. Fernando I, Cohen M, Henskens F. A systematic approach to clinical reasoning in psychiatry. Australas Psychiatry. 2013;21(3):224-230.

6. Edwards I, Jones M, Carr J, Braunack-Mayer A, Jensen GM. Clinical reasoning strategies in physical therapy. Phys Ther. 2004;84(4):312-335.

7. Jonassen DH. Toward a design theory of problem solving. Educ Technol Res Dev. 2000;48(4):63-85.

8. Jonassen D, Tessmer M. An outcomes-based taxonomy for the design, evaluation, and research of instructional systems. Train Res J. 1996;2(1996):97.

9. Rumelhart DE, Ortony A. The representation of knowledge in memory. In: Anderson RC, Spiro RJ, Montague RJ, Montague WE, eds. Schooling and the Acquisition of Knowledge. Hillsdale, USA: Lawrence Erlbaum Associates Inc.; 1976:99-135.

10. Jonassen DH. Learning to solve problems: a handbook for designing problem-solving learning environments. New York, USA: Routledge; 2011.

11. Jonassen DH, Hung W. Learning to troubleshoot: a new theory-based design architecture. Educ Psychol Rev. 
2006;18(1):77-114.

12. Jonassen DH, Ionas IG. Designing effective supports for causal reasoning. Educ Technol Res Dev. 2008;56(3): 287-308.

13. Noordman LG, Vonk W. Memory-based processing in understanding causal information. Discourse Process. 1998;26(2-3):191-212.

14. Richmond B. Systems thinking: critical thinking skills for the 1990s and beyond. Syst Dyn Rev. 1993;9(2):113133.

15. Rubenstein-Montano B, Liebowitz J, Buchwalter J, et al. A systems thinking framework for knowledge management. Decis Support Syst. 2001;31(1):5-16.

16. Mingers J, Rosenhead J. Problem structuring methods in action. Eur J Oper Res. 2004;152(3):530-554.

17. Anderson JR. Cognitive psychology and its implications. New York, USA: W. H. Freeman and Company; 1980.

18. Jacobs AE, Dolmans DH, Wolfhagen IH, Scherpbier AJ. Validation of a short questionnaire to assess the degree of complexity and structuredness of PBL problems. Med Educ. 2003;37(11):1001-1007.

19. Fischer R. Public relations problem solving: heuristics and expertise. J Public Relat Res. 1998;10(2):137-153.

20. Johnson-Laird PN. Mental models and probabilistic thinking. Cognition. 1994;50(1-3):189-209.

21. Anderson JR. Problem solving and learning. Am Psychol. 1993;48(1):35.

22. Newell A, Simon HA. Human problem solving. Englewood Cliffs, USA: Prentice-Hall; 1972.

23. Sweller J. Cognitive load during problem solving: effects on learning. Cogn Sci. 1988;12(2):257-285.

24. Scavarda AJ, Bouzdine-Chameeva T, Goldstein SM, Hays JM, Hill AV. A methodology for constructing collective causal maps. Decis Sci. 2006;37(2):263-283.

25. Overmars KP, Verburg PH. Comparison of a deductive and an inductive approach to specify land suitability in a spatially explicit land use model. Land Use Policy.
2007;24(3):584-599.

26. Sharma T, Tiwari N, Kelkar D. Study of difference between forward and backward reasoning. Int J Emerg Technol Adv Eng. 2012;2(10):271-273.

27. Monsen KA, Westra BL, Yu F, Ramadoss VK, Kerr MJ. Data management for intervention effectiveness research: comparing deductive and inductive approaches. Res Nurs Health. 2009;32(6):647-656.

28. Hinkelmann K. Forward chaining vs. backward chaining. http://didattica.cs.unicam.it/lib/exe/fetch.php?media=d idattica:choiceexams:kebi:ke-4_fc_vs_bc.pdf. Accessed October 1, 2018.

29. Scavarda AJ, Bouzdine-Chameeva T, Goldstein SM, Hays JM, Hill AV. A review of the causal mapping practice and research literature. Paper presented at: Second World Conference on POM and 15th Annual POM Conference; April 30-May 3, 2004; Cancun, Mexico. https://www. pomsmeetings.org/ConfProceedings/002/POMS_CD/Br owse\%20This\%20CD/PAPERS/002-0256.pdf. Accessed November 13, 2018.

30. Eseryel D, Ifenthaler D, Ge X. Validation study of a method for assessing complex ill-structured problem solving by using causal representations. Educ Technol Res Dev. 2013;61(3):443-463.

31. Hong L, Chijun Z, Xuemei G, Shan G, Chongde L. The influence of complexity and reasoning direction on children's causal reasoning. Cogn Dev. 2005;20(1): 87-101.

32. Sweller J, Clark RE, Kirschner PA. Teaching general problem solving does not lead to mathematical skills or knowledge. EMS Newsl. 201 1;41-42.

33. Frensch PA, Funke J. Definitions, traditions, and a general framework for understanding complex problem solving. In: Frensch PA, Funke J, eds. Complex Problem Solving: The European Perspective. Hillsdale, USA: Lawrence Erlbaum Associates Inc.; 1995:3-26.

34. Smith MU. Toward a unified theory of problem solving: 
views from the content domains. Abingdon, UK:

Routledge; 2012.

35. Larkin J, McDermott J, Simon DP, Simon HA. Expert and novice performance in solving physics problems. Science. 1980;208(4450):1335-1342.

36. Coderre S, Mandin H, Harasym PH, Fick GH. Diagnostic reasoning strategies and diagnostic success. Med Educ. 2003;37(8):695-703.

37. Kyriacou DN. Evidence-based medical decision making: deductive versus inductive logical thinking. Acad Emerg Med. 2004;11(6):670-671.

38. Harasym PH, Tsai TC, Hemmati P. Current trends in developing medical students' critical thinking abilities. Kaohsiung J Med Sci. 2008;24(7):341-355.

39. Vertue FM, Haig BD. An abductive perspective on clinical reasoning and case formulation. J Clin Psychol. 2008;64(9):1046-1068.

40. Tavakol M, Sandars J. Quantitative and qualitative methods in medical education research: AMEE guide no 90: part I. Med Teach. 2014;36(9):746-756.
41. Klein GA. A recognition-primed decision (RPD) model of rapid decision making. New York, USA: Ablex Publishing Corp.; 1993.

42. Klein G. Developing expertise in decision making. Think Reason. 1997;3(4):337-352.

43. Croskerry P. Clinical cognition and diagnostic error: applications of a dual process model of reasoning. Adv Health Sci Educ Theory Pract. 2009; 14 Suppl 1:27-35.

44. Croskerry P. A universal model of diagnostic reasoning. Acad Med. 2009;84(8):1022-1028.

45. Croskerry P. Context is everything or how could I have been that stupid. Healthc Q. 2009;12(Suppl):el71-el77.

46. May S, Withers S, Reeve S, Greasley A. Limited clinical reasoning skills used by novice physiotherapists when involved in the assessment and management of patients with shoulder problems: a qualitative study. J Man Manip Ther. 2010;18(2):84-88.

47. Pinnock R, Welch P. Learning clinical reasoning. J Paediatr Child Health. 2014;50(4):253-257. 